\title{
Increased expression of dedicator- cytokinesis-10, caspase-2 and Synaptotagmin-like 2 is associated with clinical disease activity in multiple sclerosis
}

Ion Agirrezabal', Ricardo Palacios', Beatriz Moreno ${ }^{1}$, Jorge Sepulcre², Alice Abernathy', Albert Saiz ', Sara Llufriu', Manuel Comabella ${ }^{3}$, Xavier Montalban ${ }^{3}$, Antonio Martinez ${ }^{4}$, David Arteta ${ }^{4}$ and Pablo Villoslada ${ }^{1,5^{*}}$

\begin{abstract}
Background: We aim to identify differentially expressed genes (DEGS) and its pathways associated with clinical activity of relapsing-remitting Multiple Sclerosis (RRMS).

Methods: We screened DEG in blood samples from patients with clinically stable or active RRMS ( $\geq 2$ relapses or increase in $\geq 1$ point in the EDSS (due to relapses) in 2 years follow-up), and healthy controls using DNA arrays. The DEGs identified were validated by RT-PCR in a prospective cohort of MS patients. We used Gene Ontology (GO) analysis for identifying the associated pathways and Jaspar database for identifying the associated transcriptions factors.
\end{abstract}

Results: We identified 45 DEG between the three groups (stable RRMS, active RRMS and control), being 14 of them significantly different between stable and active RRMS. We validated 14 out of the 45 DEG in the second cohort, eight out of the 14 being differentially expressed between active and stable patients (ARHGEF7, CASP2, DOCK10, DSP, ITPR1, KLDHC5, RBBP4, SYTL2). We found an overrepresentation of several pathways associated with lymphocyte activation. The analysis of regulatory networks identified the gene triplet of Dedicator of Cytokinesis-10 (DOCK10) - Caspase-2 (CASP2) - Synaptotagmin-like 2 (SYTL2) as being co-regulated by common transcription factors, pointing to lymphocyte activation pathways associated with disease activity.

Conclusions: We describe the triplet DOCK10 - CASP2 - SYTL2 as associated with the clinical activity of RRMS that suggest the role of lymphocyte activation, type 1 interferon and MAPkinase pathways in driving the presence of new relapses and disability accumulation.

Keywords: Multiple sclerosis, Biomarker, Disease activity, EDSS, Relapse rate, Gene expression pattern, Differentially expressed gene, DOCK10, CASP2, SYTL2, T cell activation, B cell activation

\footnotetext{
* Correspondence: pvilloslada@clinic.ub.es

${ }^{1}$ Center of Neuroimmunology, Institut d'Investigacions Biomèdiques August

Pi i Sunyer (IDIBAPS) - Hospital Clinic of Barcelona, Barcelona, Spain

${ }^{5}$ University of California, San Francisco, USA

Full list of author information is available at the end of the article
} 


\section{Background}

Multiple sclerosis (MS) is a clinically heterogeneous disease with a largely unpredictable course in individual patients. Clinical management of MS is hampered by the difficulty in obtaining an accurate prognosis. To achieve an accurate prognosis during the early or mid-stages of the disease, and to monitor both disease course and the response to therapy, it is essential to identify clinical or biological markers that can serve as surrogate end-points of the phenotype [1-4]. Such biomarkers would also greatly facilitate the design and monitoring of clinical trials to test new disease-modifying drugs by identifying the most appropriate patient subgroups for a specific therapy of interest.

The activity of relapsing-remitting MS (RRMS) is defined by the presence of new clinical relapses, presence of new lesions in the MRI, or increase in disability due to such relapses. The underlying pathogenesis of RRMS activity is therefore dependent on the presence of new inflammatory plaques within the brain parenchyma, as a result of the activation of the immune system. At present, it is not well known which pathways are activated during relapses and which ones are critical for defining a more active disease activity [5]. Previous gene expression studies have identified several genes such as GPR3, NFKB, SOCS3, STAT3, STAT1, CX3CR1, IDO, SLC9A9, HO-1 among others, associated with a more active disease [6-16]. However, at present none of such genes have been validated as a known biomarker of disease activity in MS and also, the pathways driving a more active disease are not well known $[2,5]$.

In the present study, we sought to identify gene signature patterns and pathways associated with RRMS and associated with clinical activity (relapses and disability worsening). Although clinical activity was defined prospectively, our study did not included MRI assessment, limiting the definition of disease activity and the opportunity to relate findings with imaging markers. We first screened gene expression from blood using DNA arrays to identify gene expression patterns that distinguish between clinically stable and active disease. Then, we validated these genes by RT-PCR in an independent prospective cohort of RRMS patients. Finally, we analyzed the regulatory network of validated genes, identifying a gene triplet composed by DOCK10, CASP2 and SYTL2 that pointed to interferon and $\mathrm{T}$ and $\mathrm{B}$ cell activation pathways as associated with disease activity in MS.

\section{Methods}

\section{Subjects}

We recruited two cohorts of patients with RRMS defined using the 2005 criteria [17]. Patients were classified as having clinically stable or active disease using following definitions: 1) stable disease - no relapses and no changes in the EDSS score during 2 years of follow-up; 2) active disease - 2 or more relapses or a 1-point increase in the EDSS score due to relapses during 2 years of follow-up. The screening cohort contained RRMS patients in the early to mid phase of the disease: stable MS patients $(n=3$; sex: $1 \mathrm{M} / 2 \mathrm{~F}$; age: $38 \pm 6$ years; disease duration: $7 \pm 0.8$ years; relapse rate during the 2 years follow-up: 0; EDSS: 0 [range: $0-1])$; active MS patients $(n=3$; sex: $1 \mathrm{M} / 2 \mathrm{~F}$; age: $33 \pm$ 7 years; disease duration: $3 \pm 0.5$ years; relapse rate over the 2 years follow-up: $2.5 \pm 0.5$, EDSS: 3 [range: 2-4]); and healthy controls ( $n=3$; sex: $1 \mathrm{M} / 2 \mathrm{~F}$; age: 36$)$. None of the patients in the screening cohort were treated with disease modifying therapies at the time of the study or in the previous month. The validation cohort was a longitudinal prospective cohort of RRMS, including stable MS patients ( $n=20$; sex: $5 \mathrm{M} / 15 \mathrm{~F}$; age: $34.0 \pm 5.3$ years; disease duration: $2.6 \pm 2.3$; relapses follow-up: 0; EDSS: $0.6 \pm 0.7$ ), active MS patients $(n=20$; sex: $10 \mathrm{M} / 10 \mathrm{~F}$; age: $30.5 \pm 4.5$; disease duration: $9.7 \pm 6.6$ years; relapse rate 2 years followup: $2.0 \pm 0.5$; EDSS: $3.8 \pm 1.4$ ) and sex and age matched healthy controls ( $n=20$; sex: $8 \mathrm{M} / 12 \mathrm{~F}$; age: $32.5 \pm 9.6$ years). Use of DMTs (Interferon-beta or Glatiramer acetate) were allowed in the validation cohort, and $33 \%$ of patients were receiving either therapy. Definition of clinical disease activity in this validation cohort was based in longitudinal prospective data and disability worsening was confirmed 6 months apart.

\section{Ethics, consent and permissions}

This study was approved by the Ethics committee of the Hospital Clinic of Barcelona. Patients were invited to participate by their neurologist after signing informed consent.

\section{RNA extraction and DNA arrays}

RNA was obtained from whole blood in the screening cohort using the PAXgene ${ }^{\mathrm{TM}}$ Blood RNA Kit (Quiagen). For the validation cohort, RNA was obtained from PBMCs purified using the Ficoll-Paque gradient system (Pharmacia Biotech). PBMCs were stored in RNAlater stabilization solution (Applied Biosystems) at $-80{ }^{\circ} \mathrm{C}$ until RNA extraction was performed. RNA was purified using the RNeasy Mini Kit and digested with the RNase Free DNase Set (both from Qiagen). The quality and quantity of the RNA was determined using a NanoDrop 2000 spectrophotometer and its integrity assessed using a 2100 bioanalyzer (Agilent Technologies). For DNA array analysis, RNA (6 $\mu \mathrm{g})$ was transcribed to cDNA using the SuperScript Choice System (Life Technologies) according to the Affymetrix Expression Analysis Technical Manual. Subsequently, cRNA was synthesized using the BioArray HighYield RNA Transcript Labeling kit (Enzo), purified with the Kit Clean-up module (Affymetrix) and finally hybridized to the HG-U133 Plus 2.0 DNA array (Affymetrix). For the validation and treatment cohorts, cDNA synthesis was performed from total RNA using the High-Capacity cDNA Archive Kit 
(Applied Biosystems). Raw DNA array data was uploaded to the ArrayExpress database (accession code E-GEOD2012082010000219) (Additional file 1).

\section{Real time PCR}

RT-PCR was performed using Low Density Arrays (LDA: Applied Biosystems) that were designed selecting TaqMan assays provided by Applied Biosystems for 45 genes plus five housekeeping genes using the following criteria: 1) minimal distance between the Affymetrix probe set and the Applied Biosystems probe set; 2) no genomic DNA detection (Additional file 2). All samples were analyzed in duplicate (384 wells) and the arrays were analyzed in triplicate. RT-PCR was carried out in the 7900 Fast RealTime PCR system using TaqMan Gene Expression Master Mix kit (both from Applied Biosystems) as follows: $2 \mathrm{~min}$ at $50{ }^{\circ} \mathrm{C} ; 10 \mathrm{~min}$ at $94.5^{\circ} \mathrm{C} ; 40$ cycles of $30 \mathrm{~s}$ at $97{ }^{\circ} \mathrm{C}$, $1 \mathrm{~min}$ at $59.7^{\circ} \mathrm{C}$ and $4{ }^{\circ} \mathrm{C}$ indefinitely [18]. SDS 2.2 .1 software was used to analyze migration on microfluid plates.

\section{Western blot analysis}

Protein levels of DOCK10, CASP2 and SYTL2 were measured by Western blots from brain tissue as previously described [6]. Antibodies used for Western blot were as follows: mouse anti-DOCK10 (Novus Biologicals), mouse

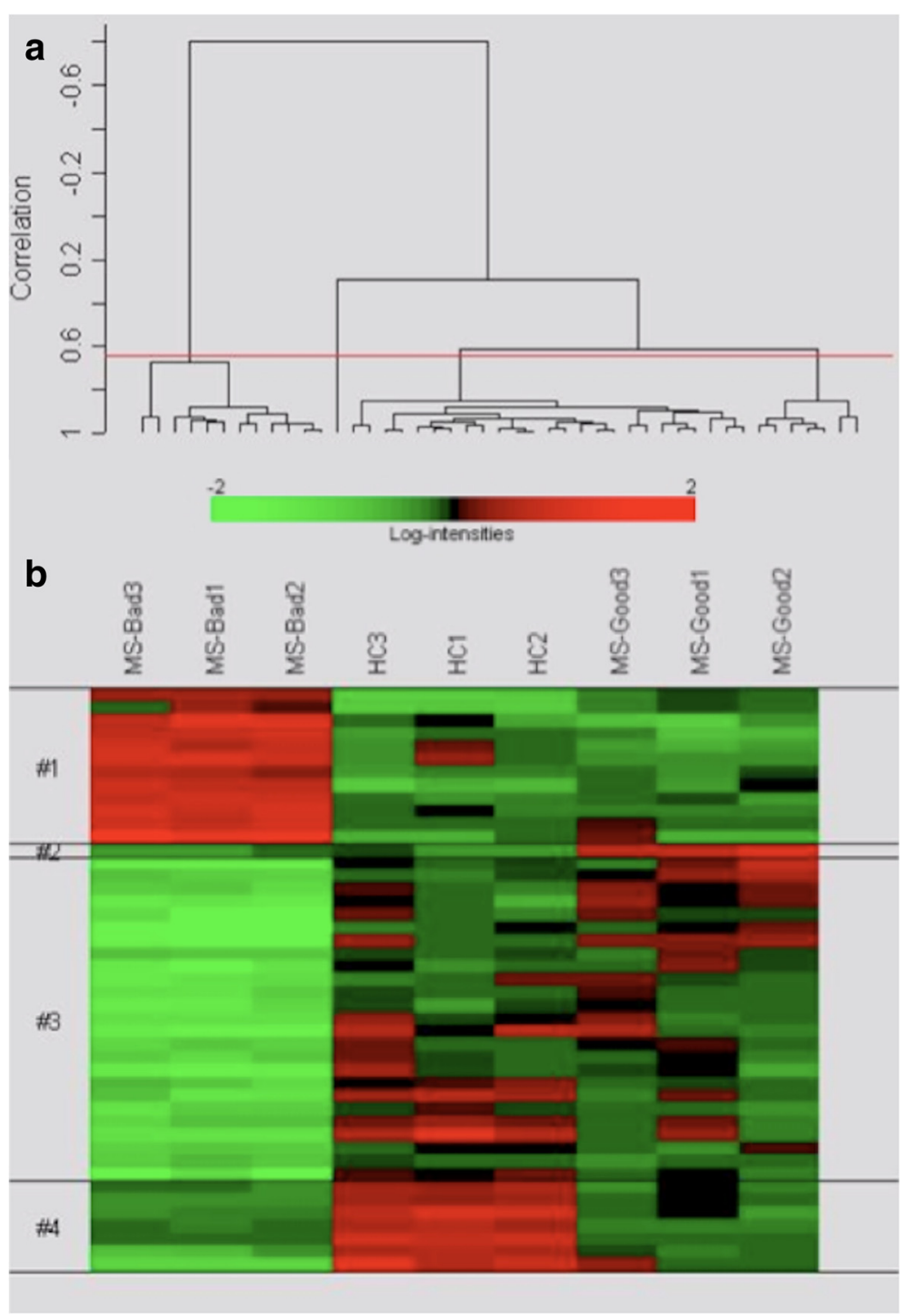

Fig. 1 Screening for differentially expressed genes in controls and in patients with clinically stable or active RRMS. 45 genes differentially expressed between all 3 conditions in patients with stable MS (MS-good), active MS (MS-bad) and controls (HC) were found. a Cluster analysis identified 4 clusters of genes using as a cut-off a correlation of 0.65 ; $\mathbf{b}$ shows the heat map of the 45 DEG grouped in the 4 clusters indentified (F-test after FDR correction) 
Table 1 Differentially expressed genes between clinically stable MS, active MS and controls by DNA array analysis (Additional file 1)

\begin{tabular}{|c|c|c|c|}
\hline Cluster & Gene symbol & $p$-value & Description \\
\hline \multirow[t]{12}{*}{ Cluster \#1 } & TNPO1 & $2.00 \mathrm{E}-07$ & Transportin 1 \\
\hline & CXCR3 & 0.0009282 & Chemokine (C-X-C motif) receptor 3 \\
\hline & ATP9A & 0.0007041 & ATPase, Class II, type 9A \\
\hline & CASP2 & 0.0003848 & Caspase 2 \\
\hline & PRX & 0.0009587 & Periaxin \\
\hline & DSP & 0.0006427 & Desmoplakin \\
\hline & MALAT1 & 0.0009593 & $\begin{array}{l}\text { Metastasis associated lung adenocarcinoma } \\
\text { transcript 1; PRO1073 }\end{array}$ \\
\hline & CXorf56 & 0.0001169 & $\begin{array}{l}\text { chromosome X open reading frame } 56 \text {, } \\
\text { hypothetical protein FLJ22965 }\end{array}$ \\
\hline & SSBP4 & 2.07E-05 & single stranded DNA binding protein 4 \\
\hline & \multirow[t]{2}{*}{ ССТ8 } & $2.13 \mathrm{E}-05$ & Chaperonin containing TCP1, subunit 8 (theta) \\
\hline & & $9.61 \mathrm{E}-05$ & Homo sapiens cDNA clone IMAGE:4824925 \\
\hline & ELSPBP1 & 0.0006039 & Epididymal sperm binding protein 1 \\
\hline Cluster \#2 & DOCK10 & 0.0003135 & Dedicator of cytokinesis 10 \\
\hline \multirow[t]{23}{*}{ Cluster \#3 } & ZNF75 & 0.0005587 & Zinc finger protein 75 (D8C6) \\
\hline & MRS2L & 0.0007168 & $\begin{array}{l}\text { MRS2-like, magnesium homeostasis factor } \\
\text { (S. cerevisiae) }\end{array}$ \\
\hline & \multirow[t]{2}{*}{ TTC10 } & 0.0006527 & $\begin{array}{l}\text { Tetratricopeptide repeat domain 10, IFT88: } \\
\text { intraflagellar transport } 88\end{array}$ \\
\hline & & 0.0002011 & Homo sapiens cDNA clone IMAGE:2348094 3 \\
\hline & MIA3 & 0.0004529 & $\begin{array}{l}\text { Melanoma inhibitory activity family, member } 3 \text {, } \\
\text { C219-reactive peptide }\end{array}$ \\
\hline & $\mathrm{KMO}$ & 7.40E-06 & $\begin{array}{l}\text { Kynurenine 3-monooxygenase (kynurenine } \\
\text { 3-hydroxylase) }\end{array}$ \\
\hline & FREB & 1.19E-05 & Fc receptor homolog expressed in B cells; FCRLA \\
\hline & MRPL16 & 0.0005844 & Mitochondrial ribosomal protein L16 \\
\hline & RNFT1 & 0.0004832 & $\begin{array}{l}\text { ring finger protein, transmembrane 1; PTD016 } \\
\text { protein }\end{array}$ \\
\hline & PRO1693 & 0.0002505 & Homo sapiens PRO1693 protein \\
\hline & ANXA4 & 0.0002741 & Annexin A4 \\
\hline & MTM1 & 0.0004095 & Myotubularin 1 \\
\hline & KLHDC5 & 0.0004232 & Kelch domain containing 5; KIAA1340 protein \\
\hline & METTL21A & 0.0004554 & Methyltransferase like 21A \\
\hline & RBBP4 & 0.0003732 & Retinoblastoma binding protein 4 \\
\hline & PPP2CB & 0.0008942 & $\begin{array}{l}\text { Protein phosphatase } 2 \text {, catalytic subunit, } \\
\text { beta isoform }\end{array}$ \\
\hline & TRAPPC11 & 0.0003224 & $\begin{array}{l}\text { Trafficking protein particle complex subunit } \\
11 \text { isoform a }\end{array}$ \\
\hline & SLC7A7 & 0.0008726 & $\begin{array}{l}\text { Solute carrier family } 7 \text { (cationic amino acid } \\
\text { transporter, } y+\text { system) }\end{array}$ \\
\hline & ALG13 & 0.0005845 & $\begin{array}{l}\text { Asparagine-linked glycosylation } 13 \text { homolog; } \\
\text { chromosome X ORF45 }\end{array}$ \\
\hline & PDIA3 & 0.000489 & $\begin{array}{l}\text { Protein disulfide isomerase family } \mathrm{A} \text {; glucose } \\
\text { regulated protein, } 58 \mathrm{kDa}\end{array}$ \\
\hline & INPP4A & 0.0007406 & $\begin{array}{l}\text { Inositol polyphosphate-4-phosphatase, type I, } \\
107 \mathrm{kDa}\end{array}$ \\
\hline & TFEC & 0.0002318 & Transcription factor EC \\
\hline & DDX23 & 0.0006295 & DEAD (Asp-Glu-Ala-Asp) box polypeptide 23 \\
\hline
\end{tabular}


Table 1 Differentially expressed genes between clinically stable MS, active MS and controls by DNA array analysis (Additional file 1) (Continued)

\begin{tabular}{|c|c|c|c|}
\hline & KIFAP3 & 0.0003059 & Kinesin-associated protein 3 \\
\hline & NEK4 & 0.0002713 & NIMA (never in mitosis gene a)-related kinase 4 \\
\hline \multirow[t]{7}{*}{ Cluster \#4 } & ZNF24 & 0.0009911 & Zinc finger protein 24 (KOX 17) \\
\hline & BTBD7 & $6.14 \mathrm{E}-05$ & BTB (POZ) domain containing 7 \\
\hline & ITPR1 & 0.0003003 & Inositol 1,4,5-triphosphate receptor, type 1 \\
\hline & WDR20 & 1.07E-05 & WD repeat domain 20 \\
\hline & LOC100507376 & 3.00E-07 & CDNA FLJ90295 fis, clone NT2RP2000240. \\
\hline & SYTL2 & 0.0002614 & Synaptotagmin-like 2 \\
\hline & ARHGEF7 & 0.0003018 & Rho guanine nucleotide exchange factor (GEF) 7 \\
\hline
\end{tabular}

We identified 45 DEGs between stable MS, active MS and healthy controls (F-test, FDR corrected $p$-value). Cluster analysis grouped the DEG in 4 clusters. Genes validated in the second cohort are highlighted in bold

anti-CASP2 and mouse anti-SYTL2 (both from Santa Cruz).

\section{Bioinformatics analysis}

In order to estimate the power of our analysis, we assumed that $95 \%$ of the evaluated probes were not differentially expressed. We aimed to detect an isolated mean difference of 1 in log-expression between groups, and set to control the rate of false positives at $10 \%$. Assuming a standard deviation of 0.5 in the difference in log-expression between groups, the power of our analysis was 0.65 . Therefore, it could be expected that $65 \%$ of genes that showed a twofold differential expression between any of the groups would be identified.

DNA array results were normalized using Microarray Suite 5.0 (MAS 5.0; Affymetrix ${ }^{\odot}$ ) and analyzed using the Biometric Research Branch (BRB) Array Tools 3.2.3 (Dr Richard Simon and Amy Peng Lam). To filter the genes with the BRB software we used the following criteria: 1) genes with an intensity $>10$ were assigned a value of $10 ; 2$ ) genes were deleted if in the $<20 \%$ of cases the change gene expression was $<1.5$ with respect to the median, if the percentage of missing values was $>50 \%$, or if the percentage of absents was $>70 \%$. For group comparison we used the F-test and for pair group comparison we used $T$-test, with a significance threshold set to $p<0.001$. Multiple comparisons were adjusted using the false discovery method with the significance threshold was set to $p<0.05$. For LDA analysis, we first discarded samples with an $\mathrm{SD}>0.38$. The normalization factor was calculated using geNorm software (https://genorm.cmgg.be) and normalized values were transformed to a logarithmic scale. For group comparison we used the Kruskal-Wallis and for pair group comparison we used Mann-Whitney U non-parametric tests, with a significance threshold set to $p<0.01$. Multiple comparisons were performed using the Bonferroni method with the significance threshold was set to $p<0.05$. Statistical

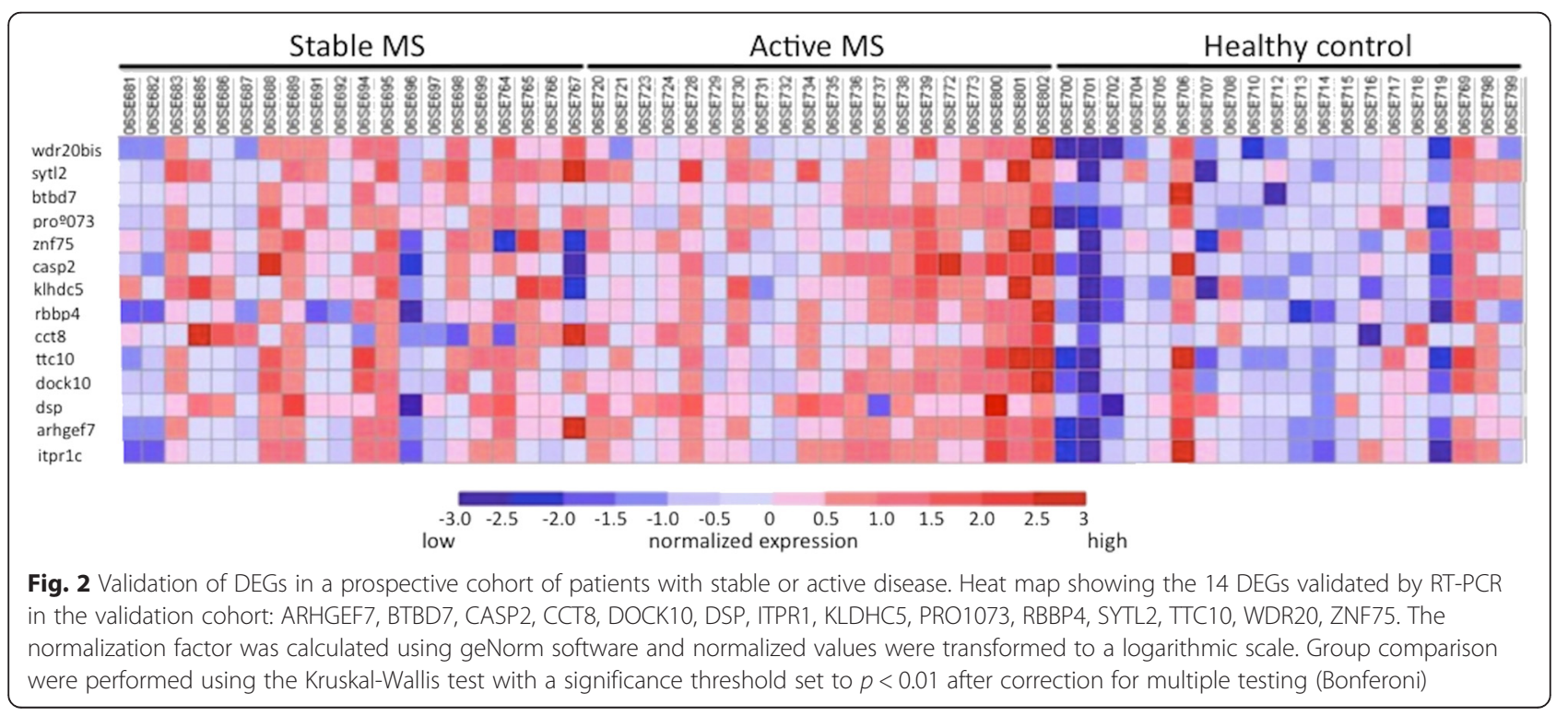


analyses were performed using SPSS 11.0 (SPSS Inc., Chicago, USA) and $\mathrm{R}$ software ( $\mathrm{R}$ Core Development Team, 2011). Correlation between DEG in the array and RT-PCR study was 0.379 . Gene Ontology was analyzed using Genecodis software (http://www.cnb.csic.es). To identify transcription factors (TF) that act as common regulators of the genes of interest, we searched for common TF binding sites based on frequency matrices obtained from the Jaspar database [19], using the position specific weight matrix (PSWM) method with both PSCAN [20] and R software.

\section{Results}

Patients with either clinically active or stable RRMS were screened, along with healthy sex- and age-matched controls. RNA extracted from whole blood was analyzed using the HG-U133 Plus 2.0 DNA array (Affymetrix), obtaining 14,705 of the 54,675 available probes which fulfilled the criteria for analysis. We performed group analysis and pairwise comparisons between each of the three groups. The group analysis identified 45 differentially expressed genes (DEGs) grouped in four clusters between the three groups (F-test, $p<0.001$, FDR correction) (Fig. 1; Table 1; Additional file 3). Genes GABPA; FREB; ZNF146; GATA3; KMO; MSH2; GP1BA; DSP; GIPC2; HAK; SSBP4; SP192; MGC35130; KIAA0826; C6orf115 significantly discriminate between patients with clinically stable and active disease ( $T$-test, $p<0.001, \mathrm{FDR}$ correction). To validate the DEGs associated with clinical activity in RRMS, we analyzed the expression of the DEGs by RT-PCR in a second independent cohort of MS patients. We determined which genes differed significantly between the three conditions, validating 14 out of the previously identified 45 DEGs that differed significantly (Kruskal-Wallis test, $p<0.01$ after Bonferroni correction for multiple testing): ARHGEF7, BTBD7, CASP2, CCT8, DOCK10, DSP, ITPR1, KLDHC5, MALAT-1 (PRO1073), RBBP4, SYTL2, IFT88 (TTC10), WDR20, ZNF75 (Fig. 2). Eight out these 14 genes, ARHGEF7, CASP2, DOCK10, DSP, ITPR1, KLDHC5, RBBP4, SYTL2, were expressed significantly different between MS patients with clinically stable or active disease (Mann-Whitney $U$ test, $p<0.01$ after Bonferroni correction for multiple testing).

In order to obtain insights about the biological role of the validated DEGs we performed Gene Ontology (GO) analysis and regulatory network analysis. The GO analysis of the DEGs revealed an overrepresentation of several pathways associated with lymphocyte activation as follows: a) GO biological process: B cell receptor signaling pathway, and positive regulation of apoptosis; b) GO molecular functions: Rho GTPase binding, Rab GTPase binding and GTPase binding; c) Enrichment analysis of KEGG pathways: cell adhesion molecules and $\mathrm{T}$ cell receptor signaling. In order to analyze the regulatory networks associated with the validated DEGs associated with disease activity, we searched for high correlation in gene expression as indication or co-regulated expression.

Among the 14 DEGs, we found only one triplet with high degree of correlation between their expression levels (by pairwise correlation). The expression of the triplet DOCK10, CASP2 and SYTL2 was highly correlated (DOCK10/CASP2: $r=0.799 ; \quad p=1.637 \mathrm{e}-11 ; \quad$ DOCK10/ SYTL2: $r=0.591 ; p=1.218 \mathrm{e}-05$; and CASP2/SYTL: $r=$ $0.613 ; p=4.506 \mathrm{e}-06$; Fig. 3 ), which would suggest the existence of master regulators for these gene triplet. The gene expression levels of the gene triplet DOCK10, CASP2

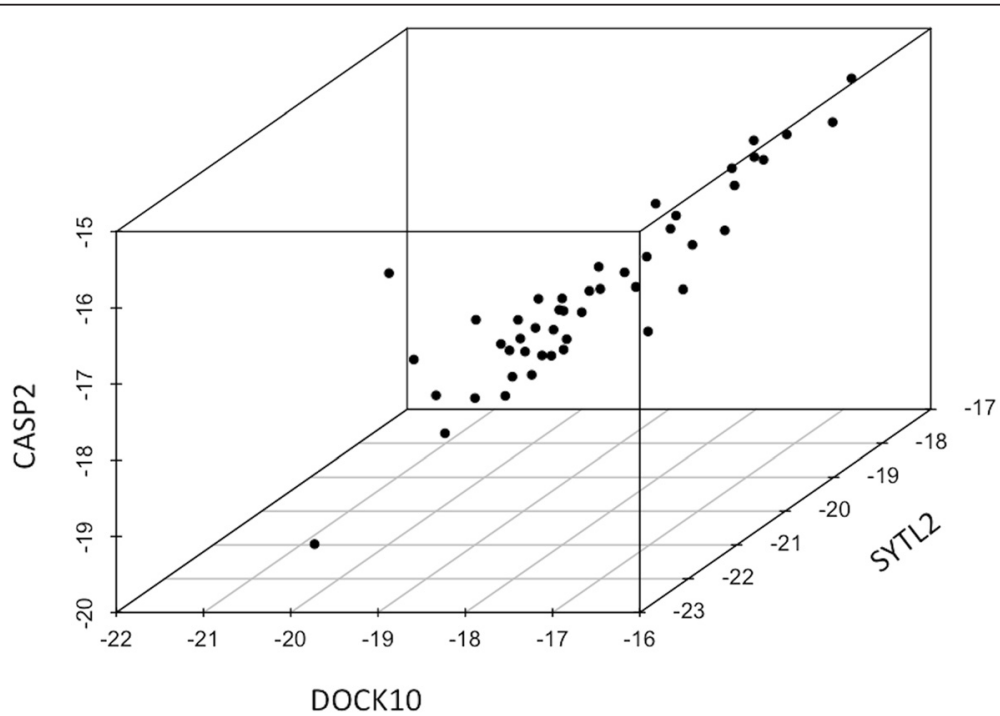

Fig. 3 Correlation of DOCK10, SYTL and CASP2 gene expression. The correlation of the gene expression levels of DOCK10, SYTL and CASP2 obtained by RT-PCR were performed using the Spearman correlation $\left(R^{2}=0.65\right)$ 
and SYTL2 were found to be increased in patients with MS (both clinically stable and active disease) compared to controls both in the DNA array as well as in the RT-PCR assays. Also, we analyzed protein levels of the triplet DOCK10, CASP2 and SYTL2 in PBMCs from MS patients and healthy controls. We observed higher levels of Dock10 and Sytl2 protein in MS patients compared with controls, and we observed a decrease in Caspase-2 in patients at protein level (Fig. 4). In order to search for common regulators of the expression of the gene triplet we performed a TF binding site prediction for DOCK10, CASP2 and SYTL2 genes. We identified 8 TF as potential master regulators: MAFB, STAT1, MYF5, FOXQ1, TCF3, SOX9, SRY and INSM1 (Table 2). A subsequent search of the Reactome and KEGG databases revealed that the top two TF MAFB and STAT1 are the downstream TF of the type I IFN and MAP kinase pathways.

\section{Discussion}

We found a gene expression signature associated with higher clinically disease activity in patients with RRMS. To identify pathways associated with such increased clinically disease activity, a bioinformatics search revealed the gene triplet DOCK10, CASP2 and SYTL2, which are activated in concert by the MAFB, STAT1 and MYF transcription factors. This pattern suggests the activation of type I IFN and MAPkinase pathways in response to immune cell receptor activation, which is in agreement with previous studies showing dysregulation of type I interferon pathway in MS $[21,22]$. In addition, $\mathrm{GO}$ analysis revealed the involvement of several pathways related with lymphocyte activation (Fig. 5).

Several gene expression patterns associated with disease activity in MS have been identified previously, implicating T-cell activation and expansion, inflammation and apoptosis/cell cycle regulation in disease activity [7-9, 13, 2326]. Biological processes that involve DEGs include the immune response, cell adhesion, cell differentiation, cellular component movement, signal transduction, blood coagulation, axon guidance, DNA and RNA transcription regulation, and the regulation of cell proliferation. Several of the TFs identified in the present study have previously been implicated in MS disease activity by the ANZ consortium [27], including TCF, MYB and the SOX family. Our findings strongly support the involvement of $\mathrm{T}$ and $\mathrm{B}$ cell activation, immune cell signaling, such as type I interferon signaling, and the regulation of cell proliferation in the pathogenesis and severity of MS. Further validation of these biomarkers in multicenter clinical studies will be required to assess its robustness in different platforms and centers.

The DOCK10/CASP2/SYTL2 gene triplet has been implicated in lymphocyte activation and function (Fig. 5). DOCK10 is a member of the dedicator of cytokinesis family, which acts as activator of the Rho family of small

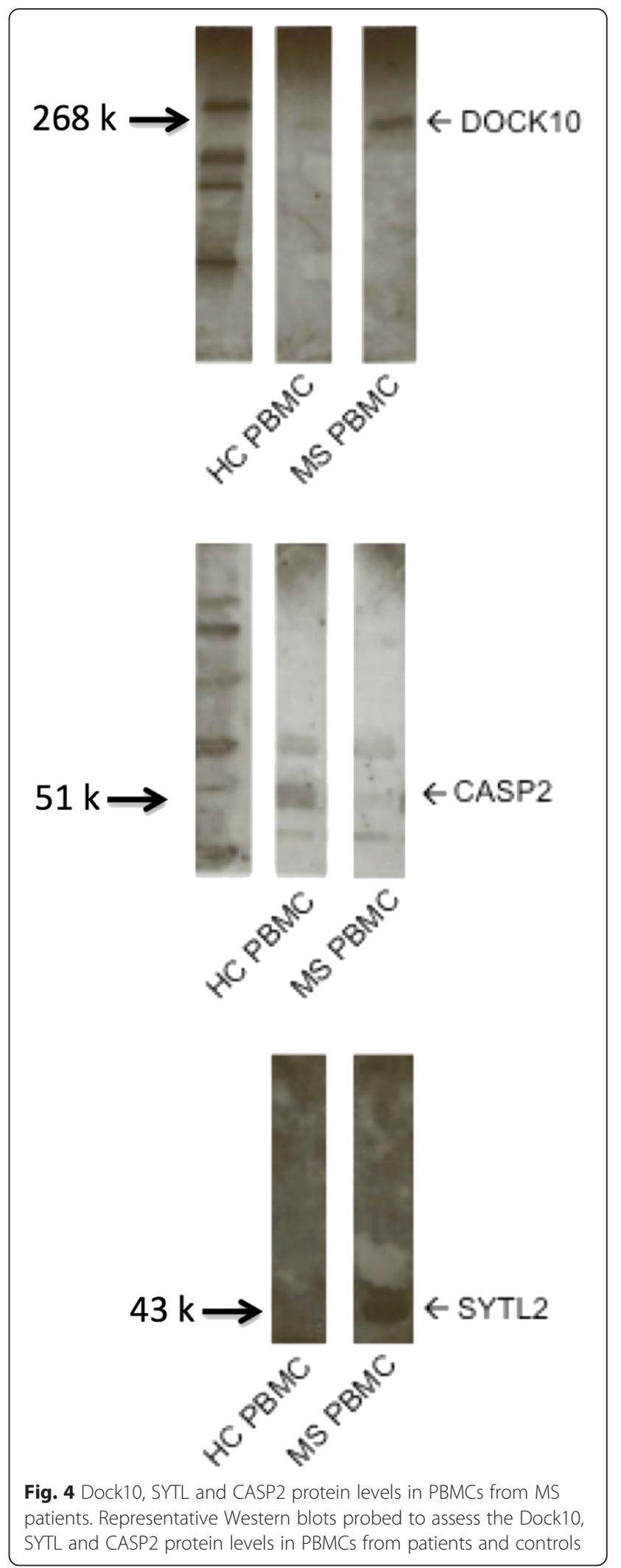


Table 2 Transcription factors associated with the MS gene expression pattern

\begin{tabular}{lll}
\hline TF & Function & $p$ \\
\hline MAFB & Transcription factor MafB & 0.000198 \\
STAT1 & Signal transducer and activator of transcription 1 & 0.001521 \\
MYF5 & Myogenic factor 5 & 0.005987 \\
TCF3 & Transcription factor E2-alpha & 0.019014 \\
SOX9 & Transcription factor SOX-9 & 0.024095 \\
SRY & Sex-determining region Y protein & 0.029105 \\
FOXQ1 & Forkhead box protein Q1 & 0.037121 \\
INSM1 & Insulinoma-associated protein 1 & 0.047677
\end{tabular}

Identification of the transcription factors (TF) associated with DOCK10, CASP2 and SYTL2 triplet, using the Jaspar database. Results are presented using the name of the TF in the Jaspar database and the $p$ value of the position-specific weight matrix analysis, ranked by the $p$ value
GTPases and mediates signaling by G-protein receptors, cytokine receptors (protein-kinases), integrins and cadherins [28]. Dock10 is mainly expressed in lymphocytes, and it is activated by the IL-4 and Rho pathways [28], exhibiting differential splicing between $\mathrm{T}$ and $\mathrm{B}$ cells [29]. Statins modulate the activity of Rho GTPases, which contributes to the induction of the Th2 phenotype and ameliorates disease severity in an animal model of MS [30]. Finally, DOCK10 is also involved in dendrite spine morphogenesis [31]. Taken together with the present results, these findings implicate DOCK-10 in increased MS disease activity through its effects on lymphocyte migration and their differentiation to the Th2 phenotype, and B cell activation.

Caspase 2 (CASP2) is a protease that is activated in response to stress (DNA damage) and it appears to participate more in the regulation of the cell cycle than in apoptosis [32]. Moreover, CASP2 acts as a tumor suppressor by inducing cell cycle arrest. CASP2 is highly

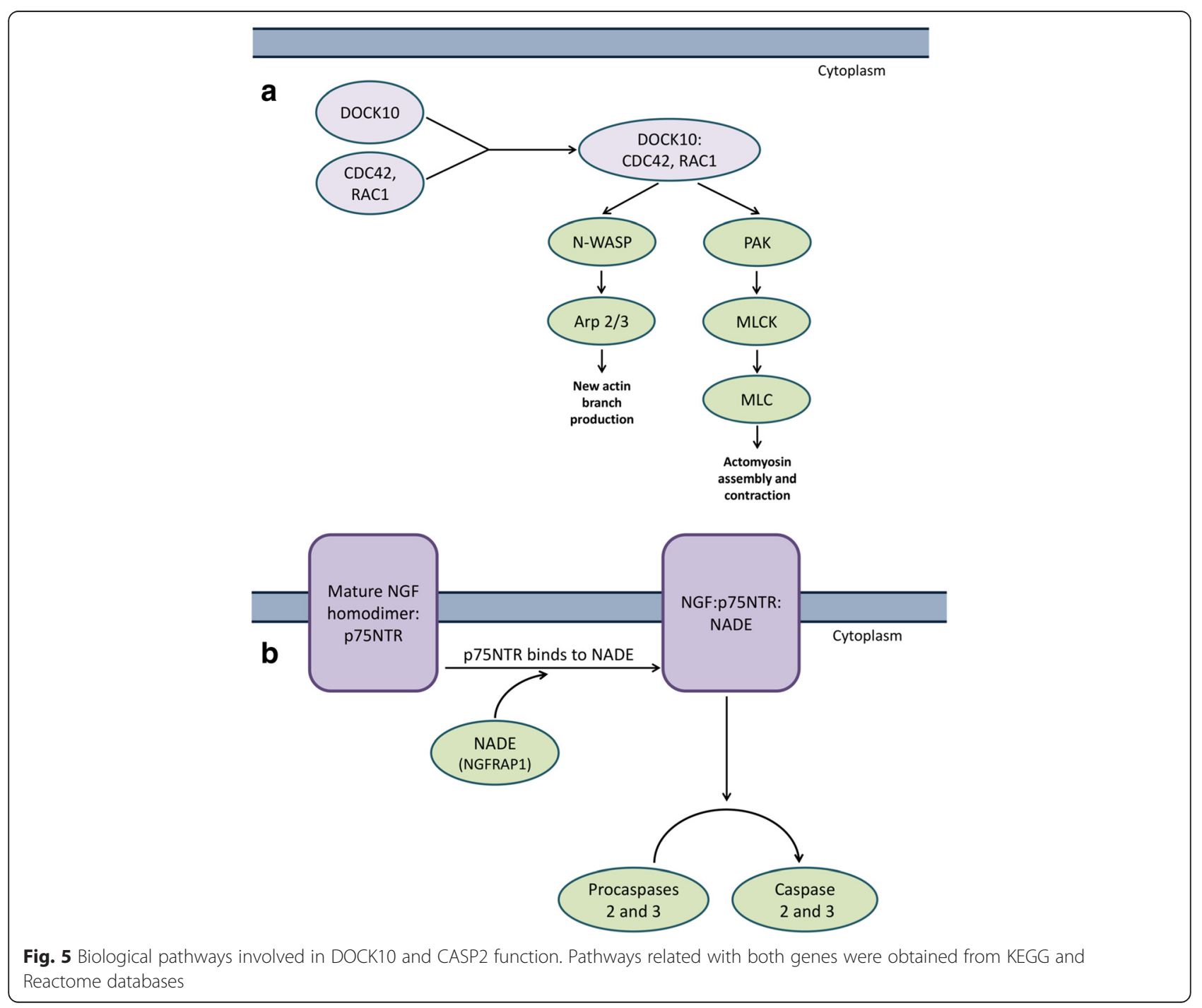


expressed in B cells during the plasmablast stage of differentiation [33], and is overexpressed in MS patients during relapses and in response to interferon-beta or intravenous immunoglobulin therapy [34-36]. In addition, CASP2 is involved specifically in apoptosis in neuronal cells $[36,37]$. Together with its modulation of the immune response, this observation strongly suggests that CASP2 contributes to the dampening of lymphocyte proliferation. We believe differences in CASP2 RNA and protein levels in our study should be related with functional regulation of this protein. One hypothesis is that the increase in protein levels may be due to higher stability or lower degradation of this protein in immune cells from MS patients. Such higher levels may suppress the levels of RNA expression as a negative feedback. Alternatively, regulation of CASP2 gene expression and efficiency to translation to protein may differ in MS patients compared to controls, probably due to pro-inflammatory state, leading to higher protein levels even with lower RNA synthesis.

Synaptotagmin-like 2 (SYTL2 or SLP2) is a member of a $\mathrm{C} 2$ domain-containing protein family and it is involved in RAB27A-dependent vesicular trafficking. Among other functions, SYTL2 participates in the maturation of immune synapses in cytotoxic $\mathrm{T}$ cells in order to promote the exocytoxis of cytotoxic granules containing perforin [38]. Interestingly, SYTL2 is required for mitochondrial fusion in response to cellular stress [39], which is critical for the restoration of mitochondrial function after stress damage [40]. Its mode of action suggests that SYTL2 participates in the stress response during inflammation.

There are several limitations of the present study that should be noted. The definitions of "active" and "stable" disease are based on the clinical expression of the disease and thus, they do not account for sub-clinical activity revealed by MRI. For this reason, our findings only apply to clinically active disease and we were not able to correlate our findings with imaging markers of disease activity. Nevertheless, misclassification of patients due to lack of MRI would increase the number of false negatives more than the number of false positives, suggesting that validated biomarkers of the proposed phenotype will persist even in the presence of the noise generated due to inaccurate clinical descriptions [3]. Although our datasets were small, we followed a sequential analysis in independent cohorts for reducing number of genes and test performed. Overall, this strategy decreased the risk of false positives, which is the main limitation in gene expression studies.

\section{Conclusion}

In summary, we describe a gene expression pattern composed by DOCK10, CASP2 and SYTL2, which is associated with a more active course of disease in patients with RRMS. The biological function of the genes and pathways identified suggest that clinical disease activity in the early to medium phase of MS is associated with increased activation of the adaptive immune system, involving both $\mathrm{T}$ and $\mathrm{B}$ cells. Additional studies will be required to further validate this molecular biomarker as well as for validating such therapeutic targets for MS and other autoimmune diseases.

\section{Additional files}

\author{
Additional file 1: Probe sets 45 DEG. (XLS $35 \mathrm{~kb}$ ) \\ Additional file 2: LDA assay design. (XLS $58 \mathrm{~kb}$ ) \\ Additional file 3: results 45 DEG from DNA arrays. (XLS $53 \mathrm{~kb}$ )
}

\section{Competing interest}

PV has received consultancy honoraria from Roche, Novartis," Digna Biotech; contract grants from Roche and Novartis and is shareholder in Bionure Farma SL. All other authors have no conflict of interest with this work.

\section{Authors' contribution}

IA, BM, RP, AA and JS carried out the RT-PCR and western-blot studies. AM and DA performed the array studies. JS, AS, SL, MC and XM contributed with samples and clinical data and classified individuals based in disease activity. IA, RP and DA performed the bioinformatic analysis. PV conceived of the study, and participated in its design and coordination and drafted the manuscript. All authors read and approved the final manuscript.

\section{Acknowledgements}

This study was supported by grants from the Spanish Ministry of Science (PSE-010000-2008-5) and Fundacion Cellex to PV, the Instituto de Salud Carlos III - Red Española de Esclerosis Multiple (RED07-0060) to PV, AS and $\mathrm{XM}$, and the 7th EU Framework Programme (Marie Curie initial training network UEPHA*MS; grant agreement $n^{\circ} 212877$ ) to PV and MC.

\section{Disclosure}

PV has received consultancy honoraria from Roche, Novartis, Medlmmune, Heidelberg Engineering, Neurotec Farma, Digna Biotech; contract grants from Roche and Novartis and is shareholder in Bionure Farma SL.

\section{Author details}

${ }^{1}$ Center of Neuroimmunology, Institut d'Investigacions Biomèdiques August Pi i Sunyer (IDIBAPS) - Hospital Clinic of Barcelona, Barcelona, Spain. ²Division of Nuclear Medicine and Molecular Imaging, Department of Radiology, Harvard Medical School, Boston, USA. ${ }^{3}$ Department of

Neurology-Neuroimmunology, Centre d'Esclerosi Múltiple de Catalunya,

Cemcat, Hospital Universitari Vall d'Hebron (HUVH), Barcelona, Spain.

${ }^{4}$ Progenica SL, Zamudio, Spain. ${ }^{5}$ University of California, San Francisco, USA.

Received: 31 August 2015 Accepted: 6 April 2016

Published online: 07 June 2016

\section{References}

1. Martin R, Bielekova B, Hohlfeld R, Utz U. Biomarkers in multiple sclerosis. Dis Markers. 2006;22(4):183-5.

2. Villoslada P. Biomarkers for multiple sclerosis. Drug News Perspect. 2010; 23(9):585-95.

3. Graber JJ, Dhib-Jalbut S. Biomarkers of disease activity in multiple sclerosis. J Neurol Sci. 2011;305(1-2):1-10.

4. Villoslada P, Baranzini S. Data integration and systems biology approaches for biomarker discovery: challenges and opportunities for multiple sclerosis. J Neuroimmunol. 2012;248(1-2):58-65.

5. Kotelnikova E, Bernardo-Faura M, Silberberg G, Kiani NA, Messinis D, Melas $I N$, et al. Signaling networks in MS: a systems-based approach to developing new pharmacological therapies. Mult Scler. 2015;21(2):138-46.

6. Moreno B, Hevia H, Santamaria M, Sepulcre J, Munoz J, Garcia-Trevijano ER, et al. Methylthioadenosine reverses brain autoimmune disease. Ann Neurol. 2006;60:323-34. 
7. Hecker M, Paap BK, Goertsches RH, Kandulski O, Fatum C, Koczan D, et al. Reassessment of blood gene expression markers for the prognosis of relapsing-remitting multiple sclerosis. PLoS One. 2011;6(12):e29648.

8. Gurevich $M$, Tuller T, Rubinstein U, Or-Bach $\mathrm{R}$, Achiron A. Prediction of acute multiple sclerosis relapses by transcription levels of peripheral blood cells. BMC Med Genomics. 2009:2:46.

9. Satoh J, Misawa T, Tabunoki H, Yamamura T. Molecular network analysis of T-cell transcriptome suggests aberrant regulation of gene expression by NFkappaB as a biomarker for relapse of multiple sclerosis. Dis Markers. 2008; 25(1):27-35.

10. Frisullo G, Mirabella M, Angelucci F, Caggiula M, Morosetti R, Sancricca C, et al. The effect of disease activity on leptin, leptin receptor and suppressor of cytokine signalling-3 expression in relapsing-remitting multiple sclerosis. J Neuroimmunol. 2007;192(1-2):174-83.

11. Frisullo G, Angelucci F, Caggiula M, Nociti V, lorio R, Patanella AK, et al. PSTAT1, PSTAT3, and T-bet expression in peripheral blood mononuclear cells from relapsing-remitting multiple sclerosis patients correlates with disease activity. J Neurosci Res. 2006;84(5):1027-36.

12. Infante-Duarte C, Weber A, Kratzschmar J, Prozorovski T, Pikol S, Hamann I, et al. Frequency of blood CX3CR1-positive natural killer cells correlates with disease activity in multiple sclerosis patients. FASEB J. 2005;19(13):1902-4.

13. Achiron A, Gurevich M, Friedman N, Kaminski N, Mandel M. Blood transcriptional signatures of multiple sclerosis: unique gene expression of disease activity. Ann Neurol. 2004;55(3):410-7.

14. Mancuso R, Hernis A, Agostini S, Rovaris M, Caputo D, Fuchs D, et al. Indoleamine 2,3 dioxygenase (IDO) expression and activity in relapsingremitting multiple sclerosis. PLoS One. 2015;10(6):e0130715.

15. Esposito F, Sorosina M, Ottoboni L, Lim ET, Replogle JM, Raj T, et al. A pharmacogenetic study implicates SLC9a9 in multiple sclerosis disease activity. Ann Neurol. 2015;78(1):115-27.

16. Fagone P, Patti F, Mangano K, Mammana S, Coco M, Touil-Boukoffa C, et al. Heme oxygenase-1 expression in peripheral blood mononuclear cells correlates with disease activity in multiple sclerosis. J Neuroimmunol. 2013; 261(1-2):82-6.

17. Polman CH, Reingold SC, Edan G, Filippi M, Hartung HP, Kappos L, et al. Diagnostic criteria for multiple sclerosis: 2005 revisions to the "McDonald Criteria". Ann Neurol. 2005;58(6):840-6.

18. Di Penta A, Moreno B, Reix S, Fernandez-Diez B, Villanueva M, Errea O, et al. Oxidative stress and proinflammatory cytokines contribute to demyelination and axonal damage in a cerebellar culture model of neuroinflammation. PloSONE. 2013;8(2):e54722

19. Bryne JC, Valen E, Tang MH, Marstrand T, Winther O, da Piedade I, et al. JASPAR, the open access database of transcription factor-binding profiles: new content and tools in the 2008 update. Nucleic Acids Res. 2008; 36(Database issue):D102-6.

20. Zambelli F, Pesole G, Pavesi G. Pscan: finding over-represented transcription factor binding site motifs in sequences from co-regulated or co-expressed genes. Nucleic Acids Res. 2009;37(Web Server issue):W247-52.

21. Comabella M, Lunemann JD, Rio J, Sanchez A, Lopez C, Julia E, et al. A type I interferon signature in monocytes is associated with poor response to interferon-beta in multiple sclerosis. Brain. 2009:132(Pt 12):3353-65.

22. Baranzini SE, Mousavi P, Rio J, Caillier SJ, Stillman A, Villoslada P, et al. Transcription-based prediction of response to IFNbeta using supervised computational methods. PLoSBiol. 2005;3(1):e2.

23. Satoh J, Nakanishi M, Koike F, Onoue H, Aranami T, Yamamoto T, et al. T cell gene expression profiling identifies distinct subgroups of Japanese multiple sclerosis patients. J Neuroimmunol. 2006;174(1-2):108-18.

24. Achiron A, Gurevich M, Snir Y, Segal E, Mandel M. Zinc-ion binding and cytokine activity regulation pathways predicts outcome in relapsingremitting multiple sclerosis. Clin Exp Immunol. 2007;149(2):235-42.

25. Corvol JC, Pelletier D, Henry RG, Caillier SJ, Wang J, Pappas D, et al. Abrogation of $\mathrm{T}$ cell quiescence characterizes patients at high risk for multiple sclerosis after the initial neurological event. Proc Natl Acad Sci U S A. 2008;105(33):11839-44.

26. Ottoboni L, Keenan BT, Tamayo P, Kuchroo M, Mesirov JP, Buckle GJ, et al. An RNA profile identifies two subsets of multiple sclerosis patients differing in disease activity. Sci Transl Med. 2012;4(153):153ra31.

27. Riveros C, Mellor D, Gandhi KS, McKay FC, Cox MB, Berretta R, et al. A transcription factor map as revealed by a genome-wide gene expression analysis of whole-blood mRNA transcriptome in multiple sclerosis. PLoS One. 2010;5(12):e14176.
28. Yelo E, Bernardo MV, Gimeno L, Alcaraz-Garcia MJ, Majado MJ, Parrado A. Dock10, a novel CZH protein selectively induced by interleukin-4 in human B lymphocytes. Mol Immunol. 2008;45(12):3411-8.

29. Alcaraz-Garcia MJ, Ruiz-Lafuente N, Sebastian-Ruiz S, Majado MJ, GonzalezGarcia C, Bernardo MV, et al. Human and mouse DOCK10 splicing isoforms with alternative first coding exon usage are differentially expressed in T and B lymphocytes. Hum Immunol. 2011;72(7):531-7.

30. Paintlia AS, Paintlia MK, Singh AK, Singh I. Inhibition of rho family functions by lovastatin promotes myelin repair in ameliorating experimental autoimmune encephalomyelitis. Mol Pharmacol. 2008;73(5):1381-93.

31. Jaudon F, Raynaud F, Wehrle R, Bellanger JM, Doulazmi M, Vodjdani G, et al. The RhoGEF DOCK10 is essential for dendritic spine morphogenesis. Mol Biol Cell. 2015;26(11):2112-27.

32. Bouchier-Hayes L, Green DR. Caspase-2: the orphan caspase. Cell Death Differ. 2012:19(1):51-7.

33. Jourdan M, Reme T, Goldschmidt H, Fiol G, Pantesco V, De Vos J, et al. Gene expression of anti- and pro-apoptotic proteins in malignant and normal plasma cells. Br J Haematol. 2009;145(1):45-58.

34. Baranzini SE, Madireddy LR, Cromer A, D'Antonio M, Lehr L, Beelke M, et al. Prognostic biomarkers of IFNb therapy in multiple sclerosis patients. Mult Scler. 2015;21(7):894-904.

35. Pigard N, Elovaara I, Kuusisto $H$, Paalavuo R, Dastidar P, Zimmermann $K$, et al. Therapeutic activities of intravenous immunoglobulins in multiple sclerosis involve modulation of chemokine expression. J Neuroimmunol. 2009;209(1-2):114-20.

36. $\mathrm{Hu} H \mathrm{HI}$, Chang HH, Sun DS. Differential regulation of caspase-2 in MPP(+)induced apoptosis in primary cortical neurons. Exp Cell Res. 2015;332(1):60-6.

37. Vigneswara V, Akpan N, Berry M, Logan A, Troy CM, Ahmed Z. Combined suppression of CASP2 and CASP6 protects retinal ganglion cells from apoptosis and promotes axon regeneration through CNTF-mediated JAK STAT signalling. Brain. 2014;137(Pt 6):1656-75.

38. Menasche G, Menager MM, Lefebvre JM, Deutsch E, Athman R, Lambert N, et al. A newly identified isoform of Slp2a associates with Rab27a in cytotoxic T cells and participates to cytotoxic granule secretion. Blood. 2008; 112(13):5052-62.

39. Tondera D, Grandemange S, Jourdain A, Karbowski M, Mattenberger $Y$, Herzig S, et al. SLP-2 is required for stress-induced mitochondrial hyperfusion. EMBO J. 2009;28(11):1589-600.

40. Court FA, Coleman MP. Mitochondria as a central sensor for axonal degenerative stimuli. Trends Neurosci. 2012;35(6):364-72.

\section{Submit your next manuscript to BioMed Central and we will help you at every step:}

- We accept pre-submission inquiries

- Our selector tool helps you to find the most relevant journal

- We provide round the clock customer support

- Convenient online submission

- Thorough peer review

- Inclusion in PubMed and all major indexing services

- Maximum visibility for your research

Submit your manuscript at www.biomedcentral.com/submit
C Biomed Central 